\title{
Angular Two-Photon Interference and Angular Two-Qubit States
}

\author{
Anand Kumar Jha, ${ }^{1}$ Jonathan Leach, ${ }^{2}$ Barry Jack, ${ }^{2}$ Sonja Franke-Arnold, ${ }^{2}$ Stephen M. Barnett, ${ }^{3}$ \\ Robert W. Boyd, ${ }^{1}$ and Miles J. Padgett ${ }^{2}$ \\ ${ }^{1}$ Institute of Optics, University of Rochester, Rochester, New York 14627, USA \\ ${ }^{2}$ Department of Physics and Astronomy, SUPA, University of Glasgow, Glasgow, United Kingdom \\ ${ }^{3}$ Department of Physics, SUPA, University of Strathclyde, Glasgow, United Kingdom
}

(Received 7 August 2009; published 5 January 2010)

\begin{abstract}
Using angular-position-orbital-angular-momentum entangled photons, we study angular two-photon interference in a scheme in which entangled photons are made to pass through apertures in the form of double angular slits, and using this scheme, we demonstrate an entangled two-qubit state that is based on the angular-position correlations of entangled photons. The entanglement of the two-qubit state is quantified in terms of concurrence. These results provide an additional means for preparing entangled quantum states for use in quantum information protocols.
\end{abstract}

DOI: 10.1103/PhysRevLett.104.010501

The signal and idler photons produced by parametric down-conversion (PDC) are entangled in several different degrees of freedom including time and energy, position and momentum, and angular position and orbital angular momentum (OAM). Entanglement of the two photons in a given degree of freedom gives rise to two-photon coherence in the corresponding domain, which manifests itself as two-photon interference in that particular domain. Several two-photon interference effects have been observed in the temporal [1-5] and spatial [6-8] domains. These effects have been used to test the foundations of quantum mechanics [9-11] and are central to many applications as well [12-14].

The existence of a Fourier relationship between angular position and OAM gives rise to angular interferenceinterference in the OAM-mode distribution of a photon field when it passes through an angular aperture [15-18]. Angular Fourier relationship in the context of angularposition-OAM entanglement leads to two-photon interference in the angular domain [19-21]. In this Letter, we study angular two-photon interference in a scheme in which entangled photons are made to pass through apertures in the form of double angular slits and, using this scheme, we demonstrate an entangled two-qubit state that is based on the angular-position correlations of the downconverted photons. Entangled two-qubit states are the necessary ingredients for many quantum information protocols [12-14], and they have previously been realized by exploring the correlations of entangled photons in variables including polarization [22], time bin [4,5], frequency [23], position [7,8], transverse momentum [24,25], and OAM [19-21]; however, to date, the angular-position correlations had not been utilized. Therefore, the results presented here not only demonstrate two-photon coherence effects in the angular domain but also provide an additional means for preparing entangled quantum states.

Let us consider the situation shown in Fig. 1(a). A Gaussian pump beam produces signal and idler photons
PACS numbers: 03.67.Bg, 42.50.Ex, 42.50.Tx, 42.65.Lm

by type-I degenerate PDC with noncollinear phase matching. The state $\left|\psi_{\text {tp }}\right\rangle$ of the down-converted two-photon field is given by $[26,27]$ :

$$
\left|\psi_{\mathrm{tp}}\right\rangle=\sum_{l=-\infty}^{\infty} c_{l}|l\rangle_{s}|-l\rangle_{i}
$$

(a)

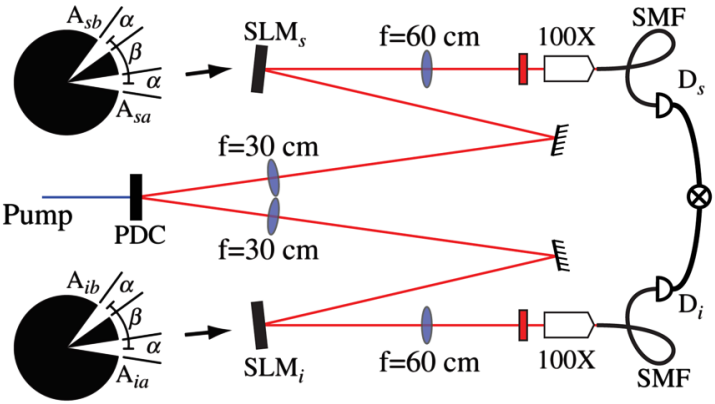

(b)

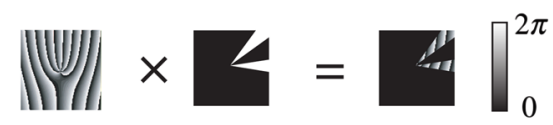

(c)

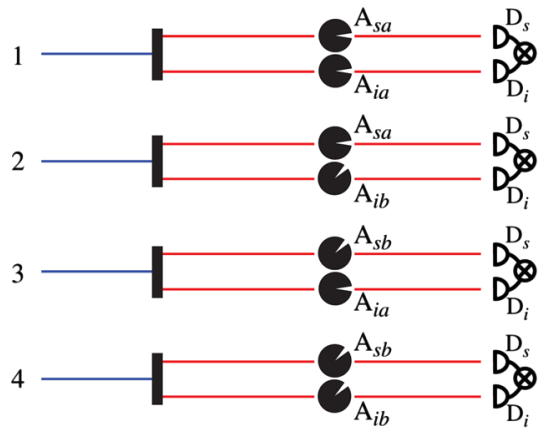

FIG. 1 (color online). (a) Schematic of the experimental setup (see text for details). (b) An example of the phase pattern impressed on the SLM. (c) Two-photon path diagrams showing the four alternative pathways by which signal and idler photons can pass through the angular slits and be detected in coincidence at detectors $D_{s}$ and $D_{i}$. 
Here $s$ and $i$ stand for signal and idler, respectively, and $|l\rangle$ represents an OAM eigenmode of order $l$, corresponding to an azimuthal phase $e^{i l \phi} .\left|c_{l}\right|^{2}$ is the probability that the signal and idler photons are generated in modes of order $l$ and $-l$, respectively. The width of this mode probability distribution is referred to as the spiral bandwidth of the two-photon field [28]. The signal and idler photons are made to pass through double angular slits [as shown in Fig. 1(a)] located in the image planes of the crystal. The amplitude transmission functions of the individual angular slits are given by

$$
\begin{gathered}
A_{j a}\left(\phi_{j}\right)=1 \text { if }-\alpha / 2<\phi_{j}<\alpha / 2 \text { or else } 0 ; \text { (2) } \\
A_{j b}\left(\phi_{j}\right)=1 \text { if } \beta-\alpha / 2<\phi_{j}<\beta+\alpha / 2 \text { or else } 0,
\end{gathered}
$$

where $j=s, i$. There are, in principle, four alternative pathways - represented by the two-photon path diagrams [3] of Fig. 1(c)-by which the down-converted photons can pass through the apertures and get detected in coincidence at detectors $D_{s}$ and $D_{i}$. In alternative 1 (4), the signal photon passes through slit $A_{s a}\left(A_{s b}\right)$ and the idler photon through slit $A_{i a}\left(A_{i b}\right)$. In alternative 2 (3), the signal photons passes through slit $A_{s a}\left(A_{s b}\right)$ and the idler photon through slit $A_{i b}\left(A_{i a}\right)$. We represent the states of the signal and idler photons in alternatives $1,2,3$, and 4 by $|s a\rangle|i a\rangle,|s a\rangle|i b\rangle$, $|s b\rangle|i a\rangle$, and $|s b\rangle|i b\rangle$, respectively. Because of the strong position correlations of the two photons in the image planes of the crystal, only alternatives 1 and 4 have appreciable probabilities. Therefore, the density matrix $\rho$ of the two-qubit state thus prepared can be written in the angularposition basis $\{|s a\rangle|i a\rangle,|s a\rangle|i b\rangle,|s b\rangle|i a\rangle,|s b\rangle|i b\rangle\}$ as:

$$
\begin{aligned}
\rho= & \rho_{11}|s a\rangle|i a\rangle\left\langle i a\left|\left\langle s a\left|+\rho_{14}\right| s a\right\rangle\right| i a\right\rangle\langle i b|\langle s b| \\
& +\rho_{41}|s b\rangle|i b\rangle\left\langle i a\left|\left\langle s a\left|+\rho_{44}\right| s b\right\rangle\right| i b\right\rangle\langle i b|\langle s b|,
\end{aligned}
$$

where $\rho_{11}$ and $\rho_{44}$ are the probabilities that the signal and idler photons are detected in alternatives 1 and 4, respectively, with $\rho_{11}+\rho_{44}=1$. The off-diagonal term $\rho_{14}$ is a measure of coherence between alternatives 1 and 4 , with $\rho_{14}=\rho_{41}^{*}$; it is, in general, a complex number and can be written as $\rho_{14}=\sqrt{\rho_{11} \rho_{44}} \mu e^{i \theta}$, where $\mu$ is the degree of coherence and $\theta$ the argument of $\rho_{14}$.

We now write the density matrix $\rho$ in the OAM basis. By taking the Fourier transforms of the amplitude transmission functions $A_{s a}\left(\phi_{s}\right)$ and $A_{i a}\left(\phi_{i}\right)[15,16]$, corresponding to each OAM mode in the summation of Eq. (1), we write $|s a\rangle|i a\rangle$ in the OAM basis as

$$
\begin{aligned}
|s a\rangle|i a\rangle= & A \sum_{l} c_{l} \sum_{l^{\prime}} \frac{1}{2 \pi} \int_{-\pi}^{\pi} d \phi_{s} A_{s a}\left(\phi_{s}\right) e^{-i\left(l^{\prime}-l\right) \phi_{s}}\left|l^{\prime}\right\rangle_{s} \\
& \times \sum_{l^{\prime \prime}} \frac{1}{2 \pi} \int_{-\pi}^{\pi} d \phi_{i} A_{i a}\left(\phi_{i}\right) e^{-i\left(l^{\prime \prime}+l\right) \phi_{i}}\left|l^{\prime \prime}\right\rangle i,
\end{aligned}
$$

where $A$ is the normalization constant to ensure that $\langle i a|\langle s a \mid s a\rangle| i a\rangle=1$. We evaluate $|s a\rangle|i a\rangle$ by substituting for $A_{s a}\left(\phi_{s}\right)$ and $A_{i a}\left(\phi_{i}\right)$ from Eq. (2). In a similar manner, we evaluate $|s b\rangle|i b\rangle$ by substituting from Eq. (3). The coincidence count rate $R_{s i}$ of detectors $D_{s}$ and $D_{i}$, which is the probability per (unit time) ${ }^{2}$ that a photon is detected at detector $D_{s}$ in mode $l_{s}$ and another at detector $D_{i}$ in mode $l_{i}$, is given by $R_{s i}={ }_{i}\left\langle\left. l_{i}\right|_{s}\left\langle l_{s}|\rho| l_{s}\right\rangle_{s} \mid l_{i}\right\rangle_{i}$. Using Eqs. (2)-(5), we find that

$$
\begin{aligned}
R_{s i}= & \frac{A^{2} \alpha^{4}}{16 \pi^{4}}\left|\sum_{l} c_{l} \operatorname{sinc}\left[\left(l_{s}-l\right) \frac{\alpha}{2}\right] \operatorname{sinc}\left[\left(l_{i}+l\right) \frac{\alpha}{2}\right]\right|^{2} \\
& \times\left\{1+2 \sqrt{\rho_{11} \rho_{44}} \mu \cos \left[\left(l_{s}+l_{i}\right) \beta+\theta\right]\right\} .
\end{aligned}
$$

The interference between the two alternatives manifests itself in the periodic dependence on the angular separation $\beta$ and on the sum of the OAMs $l_{s}+l_{i}$. From Eq. (6), ignoring the effects due to diffraction envelopes, the visibility $V$ of the coincidence fringes can be seen to be

$$
V=2 \sqrt{\rho_{11} \rho_{44}} \mu .
$$

The entanglement of a general two-qubit state can be characterized in terms of Wootters' concurrence [29,30], which ranges from 0 to 1 , with 1 corresponding to the maximally entangled two-qubit state and 0 to a nonentangled state. To calculate concurrence, we write the density matrix $\rho$ in the full $4 \times 4$ form. The concurrence $C$ is then given by $C=\max \left\{0, \lambda_{1}-\lambda_{2}-\lambda_{3}-\lambda_{4}\right\}$. Here the $\lambda_{i} \mathrm{~s}$ are the (positive) eigenvalues, in descending order, of the operator $R$ where $R^{2}=\sqrt{\rho} \sigma_{y} \otimes \sigma_{y} \rho^{*} \sigma_{y} \otimes \sigma_{y} \sqrt{\rho}$, with

$$
\sigma_{y}=\left(\begin{array}{cc}
0 & -i \\
i & 0
\end{array}\right)
$$

being the Pauli operator and $\rho^{*}$ the complex conjugate of $\rho$. For the density matrix of Eq. (4), which has only two nonzero diagonal elements, the concurrence $C$ is

$$
C=2\left|\rho_{14}\right|=2 \sqrt{\rho_{11} \rho_{44}} \mu .
$$

Comparing Eqs. (7) and (8), we see that the concurrence is equal to the visibility of angular two-photon interference fringes.

$$
C=V .
$$

In the setup of Fig. 1, the pump is a frequency-tripled, mode-locked, Nd-YAG laser (Xcyte) with a pulse repetition frequency of $100 \mathrm{MHz}$ at $355 \mathrm{~nm}$. SLM denotes a spatial light modulator from Hamamatsu, SMF a single mode fiber, and $\mathrm{F}$ an interference filter with 10-nm bandwidth, centered at $710 \mathrm{~nm}$. The $400 \mu \mathrm{m}$ diameter Gaussian pump beam was normally incident on a 3-mm-long crystal of beta barium borate, phase matched for frequency degenerate type-I down-conversion with a semicone angle of the down-converted beams of $3.5^{\circ}$. We note that for the given pump beam and phase-matching parameters, the conservation of OAM is strictly obeyed in the down-conversion process [31]. The crystal plane was imaged, with a magnification of about 5, onto the SLM planes, which were 
then imaged onto the input facets of the SMFs with a demagnification of about 380. The SLMs were used for two purposes as illustrated in Fig. 1(b). One, they were used for selecting out OAM modes [32]; and two, they were used for simulating amplitude apertures [33] described by Eqs. (2) and (3).

First of all, without any apertures, the mode probabilities $\left|c_{l}\right|^{2}$ were measured. Figure 2 shows the measured coincidence counts plotted against $l$, with signal and idler photons being detected in modes of order $l$ and $-l$, respectively. The mode probabilities $\left|c_{l}\right|^{2}$ were calculated by normalizing the counts of Fig. 2.

Second, we verify the preparation of the two-qubit state as represented by Eq. (4). Coincidence counts were measured with only one of the signal and one of the idler slits (with $\alpha=\pi / 10$ and $\beta=\pi / 4$ ) being displayed on the SLMs and with both signal and idler photons being detected in modes of order 0. Figure 3(a) shows the measured coincidence detection probabilities of the signal and idler photons in the four different alternatives. We find that the probabilities $\rho_{22}$ and $\rho_{33}$ are negligibly small, showing that the two-qubit state prepared in our experiment resembles the state represented by Eq. (4) to a very good approximation. Therefore, as shown by Eq. (9), the entanglement of the prepared two-qubit state can be characterized by measuring the visibility of two-photon interference fringes in the OAM basis.

Next, measurements were made in the OAM basis. Both signal and idler slits, with $\alpha=\pi / 10$ and $\beta=\pi / 4$, were displayed on the SLMs. SLM $i$ was adjusted to successively select out two different idler OAM modes: $l_{i}=2$ and $l_{i}=$ -2 . For each selected idler mode $l_{i}$, coincidence counts were measured as a function of the signal OAM mode $l_{s}$. Figure 3(b) shows the coincidence counts plotted against $l_{s}$ for two different values of $l_{i}$. The solid dots are theoretical

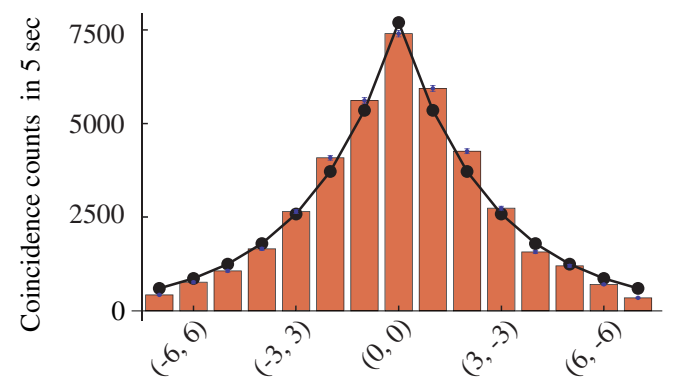

OAM-mode order of signal and idler photons $(l,-l)$

FIG. 2 (color online). Measurements of the OAM-mode probabilities with the SLMs set for uniform reflectivity. Measured coincidence counts are given as a function of $l$, the OAM-mode order of the detected signal photon, with $-l$ being the OAMmode order of the idler photon. The solid dots are the expected values based on the theoretical prediction of Ref. [28] [Eq. (10)]; the solid line through the dots is drawn as a visual guide. The fitting parameters are an overall constant factor and the effective beamwidth of down-converted modes as measured by the detection system. fits based on Eq. (6), using the values of $\left|c_{l}\right|^{2}$ calculated from Fig. 2. The visibility of the two-photon fringes is $92.8 \%$ (96.3\%, after correcting for random coincidences), within $2 \%$ experimental error. Thus, using Eq. (9), we find that the concurrence of the prepared two-qubit state is 0.928 ( 0.963 , after correcting for random coincidences).

However, as $\rho_{22}$ and $\rho_{33}$ are not precisely zero in our experiment, it is desirable to quantify the error in the above estimation of concurrence. Although a precise error calculation requires knowledge of all of the 16 different terms of the two-qubit density matrix and is beyond the scope of this Letter, a realistic estimation can be obtained by modeling the probabilities $\rho_{22}$ and $\rho_{33}$ as a small amount of noise in the two-qubit density matrix $\rho$ in Eq. (4). The corrected density matrix $\rho^{(c)}$ is then given by $\rho^{(c)}=\rho+$ $\rho_{22}|s a\rangle|i b\rangle\left\langle i b\left|\left\langle s a\left|+\rho_{33}\right| s b\right\rangle\right| i a\right\rangle\langle i a|\langle s b|$ and the visibility $V^{(c)}$ of angular two-photon fringes by $V^{(c)}=2 \sqrt{\rho_{11} \rho_{44}} \mu$, with $\rho_{11}+\rho_{22}+\rho_{33}+\rho_{44}=1$. The concurrence $C^{(c)}$ of
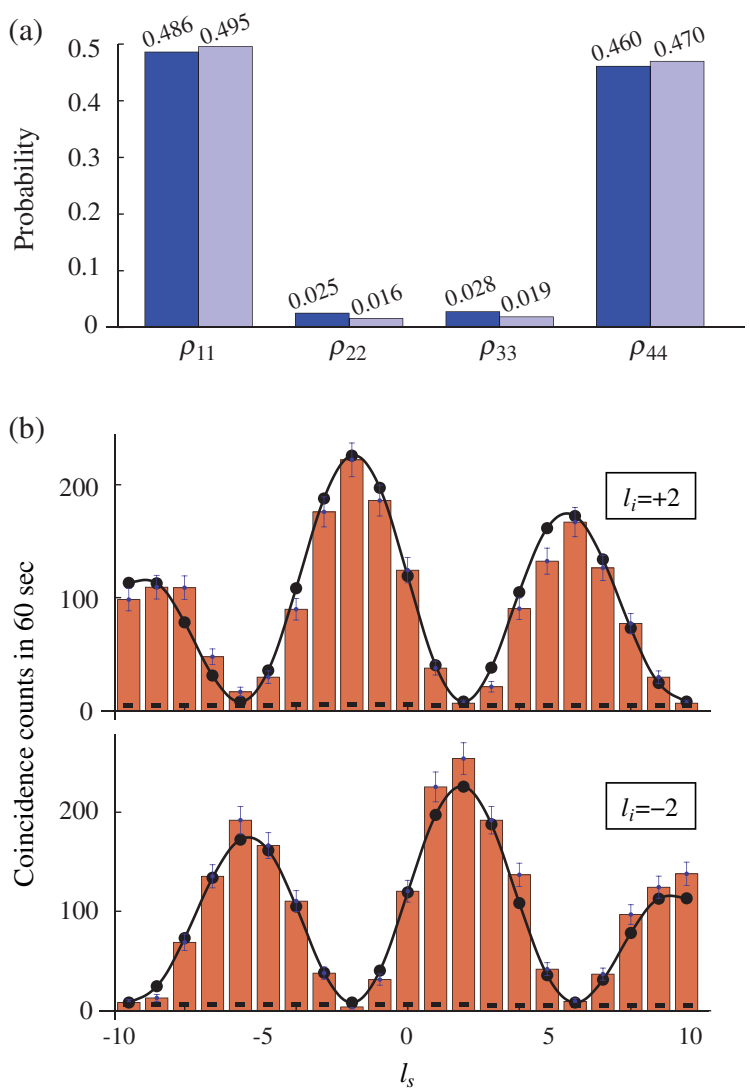

FIG. 3 (color online). (a) Measured coincidence detection probabilities $\rho_{11}, \rho_{22}, \rho_{33}$, and $\rho_{44}$. The measured probabilities are shown by blue bars (black in the printed version); the probabilities after correcting for random coincidences are shown by light blue bars (gray in the printed version). (b) Measured coincidence counts (light red, gray in the printed version) as functions of $l_{s}$ for two different values of $l_{i}$, with $\alpha=\pi / 10$ and $\beta=\pi / 4$. The solid dots are theoretical fits obtained from Eq. (6), the solid lines are visual guides, and the dashed lines are measured random coincidences for the 10-ns coincidence detection window. 

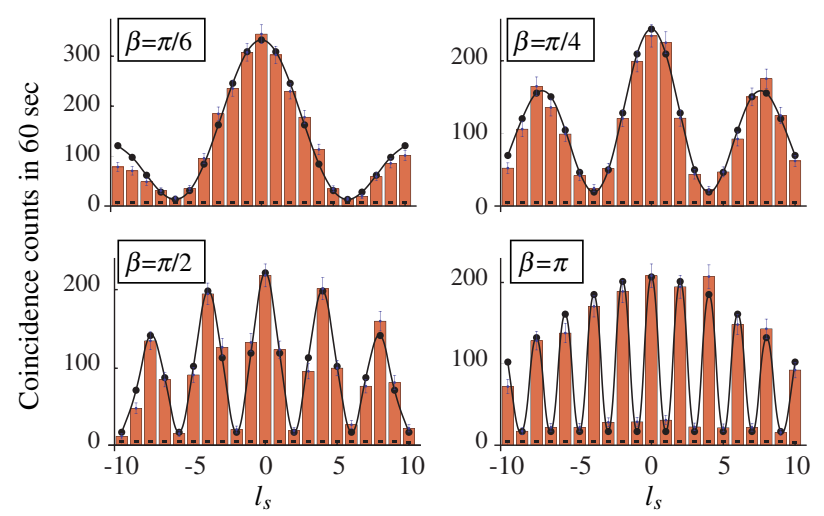

FIG. 4 (color online). Measured coincidence counts as a function of $l_{s}$ for four different values of $\beta$, with $\alpha=\pi / 10$ and $l_{i}=$ 0 . Each plot is an average of 24 different plots, taken with the starting angles of both the signal and idler apertures rotated in steps of $15^{\circ}$ from $0^{\circ}$ to $360^{\circ}$.

the state takes the following form: $C^{(c)}=2 \sqrt{\rho_{11} \rho_{44}} \mu-$ $2 \sqrt{\rho_{22} \rho_{33}}$, and with the above expression for visibility, it can be written as

$$
C^{(c)}=V^{(c)}-2 \sqrt{\rho_{22} \rho_{33}},
$$

which reduces to the formula given in Eq. (9) when $\rho_{22}=\rho_{33}=0$. Using the experimental values in Figs. 3(a) and 3(b), we find that the concurrence $C^{(c)}$ of the prepared two-qubit state is 0.875 (0.929, after correcting for random coincidences), which differs from the value obtained from Eq. (9) by about 6\% (4\% after correcting for random coincidences).

Finally, to test the applicability of our method, we perform a series of experiments with various values of slit separation $\beta$. Figure 4 shows the measured coincidence counts as a function of $l_{s}$ for four different values of $\beta$ : $\pi / 6, \pi / 4, \pi / 2$, and $\pi$. The high visibility (between $85 \%$ to $92 \%$, without correcting for random coincidences) of these plots shows that the angular-position correlations of the signal and idler photons are almost uniform over the entire range of $2 \pi$ radians and that the method is applicable over a wide range of $\beta$ values.

In conclusion, we have studied two-photon interference in the angular domain and have reported experimental demonstrations of an entangled two-qubit state that is based on the angular-position correlations of the entangled two-photon field. These results provide an additional means for preparing entangled two-qubit states and constitute a step towards better understanding angularposition-OAM entanglement [26-28] and thus towards finding novel ways of utilizing OAM basis for quantum information science [19-21,32,34]. We believe that the method presented in this Letter can be easily generalized for preparing entangled two-qudit states, using apertures with $d$ angular slits.
We gratefully acknowledge financial support through a MURI grant from the U.S. Army Research Office, through DARPA/DSO, the Future and Emerging Technologies (FET) programme within the Seventh Framework Programme of the European Commission, HIDEAS (No. FP7-ICT-221906), the UK EPSRC, RCUK, the Royal Society, and the Wolfson Foundation. We would like to thank Hamamatsu for their support of this work. We thank M. N. O'Sullivan, L. Neves, and S. Agarwal for useful discussions, and we also thank the referees of this Letter for some important comments and suggestions.

[1] C. K. Hong, Z. Y. Ou, and L. Mandel, Phys. Rev. Lett. 59, 2044 (1987).

[2] T. J. Herzog et al., Phys. Rev. Lett. 72, 629 (1994).

[3] A. K. Jha et al., Phys. Rev. A 77, 021801(R) (2008).

[4] J. Brendel et al., Phys. Rev. Lett. 82, 2594 (1999).

[5] R. T. Thew et al., Phys. Rev. A 66, 062304 (2002).

[6] E. J. S. Fonseca et al., Phys. Rev. A 61, 023801 (2000).

[7] L. Neves et al., Phys. Rev. Lett. 94, 100501 (2005).

[8] L. Neves et al., Phys. Rev. A 76, 032314 (2007).

[9] A. Aspect, P. Grangier, and G. Roger, Phys. Rev. Lett. 49, 91 (1982).

[10] L. Mandel, Rev. Mod. Phys. 71, S274 (1999).

[11] A. Zeilinger, Rev. Mod. Phys. 71, S288 (1999).

[12] A. K. Ekert, Phys. Rev. Lett. 67, 661 (1991).

[13] C. H. Bennett and S. J. Wiesner, Phys. Rev. Lett. 69, 2881 (1992).

[14] C. H. Bennett et al., Phys. Rev. Lett. 70, 1895 (1993).

[15] S. M. Barnett and D. T. Pegg, Phys. Rev. A 41, 3427 (1990).

[16] S. Franke-Arnold et al., New J. Phys. 6, 103 (2004).

[17] B. Jack, M. Padgett, and S. Franke-Arnold, New J. Phys. 10, 103013 (2008).

[18] A. K. Jha et al., Phys. Rev. A 78, 043810 (2008).

[19] A. Vaziri, G. Weihs, and A. Zeilinger, Phys. Rev. Lett. 89, 240401 (2002).

[20] N. K. Langford et al., Phys. Rev. Lett. 93, 053601 (2004).

[21] J. Leach et al., Opt. Express 17, 8287 (2009).

[22] P. G. Kwiat et al., Phys. Rev. Lett. 75, 4337 (1995).

[23] S. Ramelow et al., Phys. Rev. Lett. 103, 253601 (2009).

[24] J. G. Rarity and P. R. Tapster, Phys. Rev. Lett. 64, 2495 (1990).

[25] M. N. O’Sullivan-Hale et al., Phys. Rev. Lett. 94, 220501 (2005).

[26] S. P. Walborn et al., Phys. Rev. A 69, 023811 (2004).

[27] S. Franke-Arnold et al., Phys. Rev. A 65, 033823 (2002).

[28] J. P. Torres, A. Alexandrescu, and L. Torner, Phys. Rev. A 68, 050301(R) (2003).

[29] W. K. Wootters, Phys. Rev. Lett. 80, 2245 (1998).

[30] B. Jack et al., New J. Phys. 11, 103024 (2009).

[31] G. Molina-Terriza, J. Torres, and L. Torner, Opt. Commun. 228, 155 (2003).

[32] A. Mair et al., Nature (London) 412, 313 (2001).

[33] J. Leach et al., New J. Phys. 7, 55 (2005).

[34] G. Tyler and R. Boyd, Opt. Lett. 34, 142 (2009). 